remote Himalayan region that prevents telemetry support, rendering it unavailable. Instead, telemetry-based monitoring of the glacierized Himalayan catchment using satellite systems (e.g., the Narrowband Internet of Things) is needed to take timely actions during the next hydrological disaster. The integration of monitoring devices with satellite networks will not only provide telemetry support in remote locations that lack complete cellular connectivity but will also provide greater connectivity coverage in the cellular dead zones in extreme topographies such as valleys, cliffs, and steep slopes.

Real-time data would help to develop a strong network of early flood warning systems in the glacierized catchment of the Himalayas. Real-time monitoring technologies would not only help to predict and warn of the impending danger and prevent loss of life, but the availability of real-time data would allow scientists to monitor the performance of the installed instruments remotely and take timely actions against any instrument malfunction, preventing the loss of vital data. Therefore, these enriched datasets will help us to better understand the effects of climate change on the Third Pole, which is often regarded as a "white spot" on the global map-indicating the presence of very limited continuous field hydrometeorological data (9).

\section{REFERENCES AND NOTES}

1. P.D.A. Kraaijenbrink, M. F.P. Bierkens, A. F. Lutz, W. W. Immerzeel, Nature 549, 257 (2017).

2. A. F. Lutz, W. W. Immerzeel, A. B. Shrestha, M. F.P. Bierkens, Nat. Clim. Chang. 4,587 (2014).

3. J. M. Maurer, J. M. Schaefer, S. Rupper, A. Corley, Sci. Adv . 5, eaav7266 (2019)

4. J. M. Maurer et al., Sci. Adv. 6, eaba3645 (2020).

5. I. Haddeland et al.,Proc. NatI. Acad. Sci. U.S.A.111, 3251 (2014).

6. R. Hocket al.,"High Mountain Areas" in IPCC Special Report on the Ocean and Cryosphere in a Changing Climate (2019)

7. G. Zhang et al., Remote Sens. Environ. 237, 11154 (2020),

8. A. B. Shrestha, G. C. Ezee, R.P.Adhikary, S. K. Rai, Resource Manual on Flash Flood Risk Management. Module 3: Structural Measures [International Centre for Integrated Mountain Development (ICIMOD), 2012]

9. The Intergovernmental Panel on Climate Change, Climate Change 2007: The Physical Science Basis (Cambridge Univ. Press, 2013).

10. S. Nandargi, O. N. Dhar, Hydrol. Sci. J. 56, 930 (2011)

11. A. P. Dimrietal., Earth Sci. Rev. 53,225 (2017).

12. J. D. Ives, R. B. Shrestha, P. K. Mool, Formation of Glacial Lakes in the Hindu Kush-Himalayas and GLOF Risk Assessment (ICIMOD, 2010).

\section{ACKNOWLEDGMENTS}

This work was supported by the Department of Science and Technology (grant IUSSTF/WAQM-Water Quality Project-IIT Kanpur/2017) and the Ministry of Earth Sciences (MoES) Government of India (grant MOES/PAMC/H\&C/79/2016PC-II) to I.S.S. T.S. is thankful for a postdoctoral scholarship from the Indian Institute of Technology Kanpur. We thank V. Vinoj and K. Sri Harsha for their suggestions.

\title{
QUANTUM GASES
}

\section{The Weyl side of ultracold matter}

\section{Ultracold gases in optical lattices provide control and tunability for the exploration of Weyl semimetal physics}

\section{By Nathan Goldman ${ }^{1}$ and Tarik Yefsah ${ }^{2}$}

\section{I} he discovery of Weyl semimetals in 2015 was a breakthrough in the modern history of quantum matter, connecting relativistic phenomena predicted in particle physics with unusual topological properties of the solid state (1). This connection originates from the peculiar band structure of Weyl materials. In general, the band structure of a solid governs which energies are accessible to an electron moving with a given momentum. In Weyl semimetals, energy bands touch at singular points (the Weyl nodes), around which energy has a linear dependence on momentum $k$, reminiscent of relativistic elementary particles. On page 271 of this issue, Wang $e t$ $a l$. (2) realized Weyl-type band structures for ultracold atoms with a high degree of control and tunability. This work paves the way for the exploration of the properties of Weyltype band structures with a bottom-up, tunable approach and incremental complexity.

${ }^{1}$ Interdisciplinary Center for Nonlinear Phenomena and Complex Systems, Université Libre de Bruxelles, CP 231 Campus Plaine, B-1050 Brussels, Belgium. ² Laboratoire Kastler Brossel, ENS-Université PSL, CNRS, Sorbonne Université, Collège de France, 24 rue Lhomond 75005 Paris, France. Email: tarik.yefsah@lkb.ens.fr
Whenever a concept of relativity finds an echo in the realm of quantum materials, it triggers a wave of astonishment and excitement. Indeed, relativistic phenomena are naturally linked to high-energy physics. The excitement comes from the possibility of bringing to reality predictions that otherwise may only be recognized for their mathematical esthetics. Herman Weyl's 1929 prediction of hypothetical massless fermions is a prime example because their existence was never confirmed in particle-physics experiments but was instead observed in solid-state quantum materials (1).

Observing "pseudo-relativistic electrons" in materials is not completely surprising, given the formal equivalence between the Dirac or Weyl equations describing relativistic elementary particles and the effective Schrödinger equation describing electronic excitations in semimetals (1). Beyond this formal analogy, the pseudo-relativistic band structure of Weyl semimetals also hosts a robust mathematical property, a so-called topological defect that cannot be removed under small deformations of the crystal (1). To appreciate this notion, one should first realize that a fictitious "magnetic" field (also called Berry curvature) can be associated with the energy bands of crystalline struc-

\section{Tunable Weyl nodes for ultracold atoms}

The engineering of Weyl-type band structures in optical lattices relies on correlating the spin and momentum of the atoms along all three spatial directions. By realizing such a spin-orbit coupling (SOC), Wang et al. formed and tuned a Weyl-type band structure for ultracold rubidium atoms.
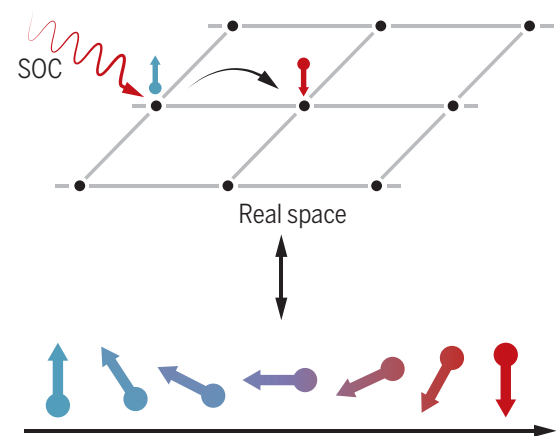

Momentum space

\section{Tuning spin-orbit coupling}

Cold atoms in two internal states (spin-up and spin-down) move on a three-dimensional (3D) optical lattice in the presence of laser-induced SOC. Tomography was used to extract the spin texture in momentum space.

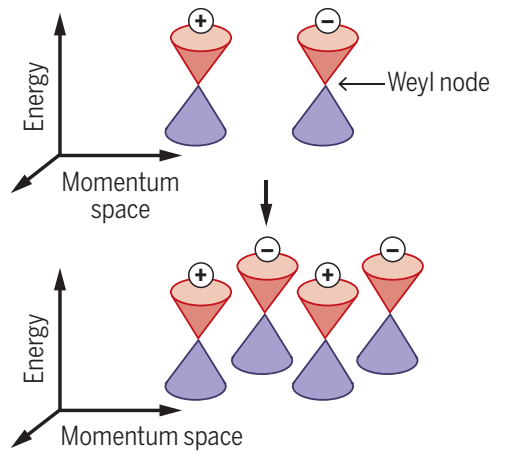

Varying Weyl points

Tuning 3D SOC modifies the band structure and creates configurations with different numbers of Weyl nodes. Nodes always come in pairs with opposite topological charges (+ and -). 
tures (3). In the vicinity of a Weyl node, this fictitious magnetic field emanates radially. This structure implies the existence of a fictitious monopole (a point-like source of fictitious magnetic field) that is located exactly at the Weyl node.

The key ingredient for the emergence of Weyl-type band structures is spin-orbit coupling (SOC) (1), which is an interplay between the intrinsic angular momentum of a particle (spin) and its trajectory. Spin-orbit coupling can create band structures with strong correlations between the momentum and spin of electrons and give rise to a variety of topological quantum states of matter (3). However, not all SOCs are equivalent: Emerging topological properties crucially depend on the dimensionality of both the SOC and the material (3). In the case of Weyl semimetals, three-dimensional (3D) SOC is required and, as is often the case in solidstate physics, the path to the 2015 discovery relied on the identification of the "right" materials. Since then, much effort has focused on finding materials displaying only a few Weyl nodes, and ideally only a single pair, the minimum number allowed by the socalled doubling theorem (1).

Synthetic lattices for ultracold atomic gases allow Weyl-type band structures to be built by putting in the right ingredients (4), rather than search for new materials. Wang et al. not only created a band structure ondemand with either one or two pairs of Weyl nodes, but they also dynamically turned a trivial band structure into a Weyl-type one. They set ultracold atomic clouds of rubidium-87 in their designed laser landscape that both provides a $3 \mathrm{D}$ lattice potential that mimics the crystalline structure of solids and couples the motion of the atoms to their spin in all three spatial directions. Its Weylsemimetal band structure was revealed by performing tomography of the 3D momentum distribution of the atoms while keeping track of their spin texture (see the figure).

With the spin-momentum distribution at hand, they probed the topological nature of the band structure; namely, the monopole charge associated with each Weyl node. The tunability of the setup allowed the effects of a sudden change in the system's parameters to be studied. Starting from atoms initially placed in a regular lattice configuration without 3D-SOC, they suddenly activated the Weyl semimetal lattice and followed the spin-population dynamics in the band structure. This provided a complementary measure of the Weyl-nodes location. Further studies with ultracold gases could explore special surface modes, previously revealed in the solid state (5) and photonics (6), whose robustness directly follows from the topological nature of the Weyl nodes. The spec- troscopic detection of these Fermi-arc states could be facilitated by confining the atoms in a box with sharp boundaries $(7,8)$.

Cold-atom realizations of Weyl semimetals could offer an ideal platform to study their exceptional transport properties. Subjecting them to synthetic electric and magnetic fields should induce a so-called chiral anomaly, a quantized transport of particles from one Weyl node to the other (1). This anomaly is subtle in the context of particle physics, but it could be directly measured in optical lattices through momentumdistribution measurements (9). The chiral anomaly can also be induced by axial gauge fields generated by modulating the optical lattice in space and time (10). Weyl semimetals also exhibit anomalous Hall and circular photogalvanic effects (1) that could both be revealed in optical-lattice setups through circular shaking (11). The tunability of this setting could be exploited to engineer various types of semimetals displaying exotic nodal lines, rings, or spheres in the band structure $(12,13)$. Synthetic 3D SOCs also constitute a central ingredient for the engineering of 3D topological insulators with cold gases (3).

Promoting a 3D optical lattice to a fictitious 4D lattice could also be envisaged through the concept of synthetic dimension (14)-for example, by inducing motion along the space spanned by atomic internal states. Applying this strategy could produce a 4D Weyl semimetal that displays fictitious KalbRamond monopole fields originally introduced in string theory (15). Another exciting possibility would be to engineer 4D topological insulators (14), which have the appealing property of displaying a single isolated Weyl node on their surfaces.

\section{REFERENCES AND NOTES}

1. N. P. Armitage, E. J. Mele, A. Vishwanath, Rev. Mod. Phys. 90,015001 (2018)

2. Z-Y.Wang etal. Science 372,271(2021).

3. X.-L. Qi, S.-C. Zhang, Rev. Mod. Phys. 83,1057 (2011).

4. N. R. Cooper, J. Dalibard, B. Spielman, Rev. Mod. Phys. 91, 015005 (2019)

5. S.-Y.Xuet al.,Science 349,613 (2015).

6. B. Yang etal., Nat. Commun .8, 97 (2017).

7. T. Dubčeket al.,Phys. Rev. Lett.114, 225301 (2015)

8. J.Y. Choietal., Science 352,1547 (2016).

9. S. Roy etal.,Phys. Rev. B 94,161107(R) (2016).

10. S. Roy etal.,2D Mater. 5, 024001 (2018).

11. D. T. Tran, A. Dauphin, A. G. Grushin, P.Zoller, N. Goldman, Sci. Adv. 3, e1701207 (2017)

12. B. Song et al., Nat. Phys. 15, 911 (2019).

13. G. Salerno, N. Goldman, G. Palumbo, Phys. Rev. Res. 2 $013224(2020)$

14. T. Ozawa, H. M. Price, Nat. Rev. Phys. 1,349 (2019).

15. G. Palumbo, N. Goldman, Phys. Rev. Lett. 121, 170401 (2018).

\section{ACKNOWLEDGMENTS}

N.G. acknowledges A. Grushin for discussions on the chiral anomaly and the Fund for Scientific Research (FRS-FNRS) (Belgium) and the European Research Council (Starting Grant TopoCold) for financial support.

10.1126/science.abg0892
NEUROSCIENCE

Mosaic synapses in
epilepsy
Mismatch of synaptic
cadherins perturbs
hippocampal circuitry

\section{By Belal Shohayeb and Helen M. Cooper}

I he inherited X-linked early-onset childhood epilepsy, called EFMR (epilepsy and mental retardation limited to females), has baffled clinicians and geneticists for more than 50 years. In contrast to other X-linked disorders in which the hemizygous (hemi) male, but not the heterozygous (het) female is affected, it is only the het females that exhibit seizures and intellectual disability $(1,2)$. Clues to the origin of this enigmatic disorder came when deleterious mutations in the $\mathrm{X}$ chromosome gene protocadherin-19 (PCDH19), which encodes a cell adhesion molecule, were identified (3). On page 255 of this issue, Hoshina et al. (4) provide answers to two pieces of the EFMR puzzle: They reveal that hippocampal synaptic transmission is compromised in Pcdh19 het female mice but not in hemi males, and they provide a molecular explanation for how the retention of one wild-type (WT) allele, but not the loss of both alleles, disrupts neuronal connectivity.

In general, females with X-linked disorders are asymptomatic because they carry one WT allele. Conversely, hemi males only express the mutated gene and are unable to produce a functional protein. What accounts for the EFMR sex reversal? Important insight came with the identification of idiopathic males with EFMR symptoms (5). Affected males carried postzygotic somatic PCDH19 mutations, and therefore regions within the developing brain that normally express $\mathrm{PCDH} 19$ comprise a mixture of PCDH19-positive and -negative (has a nonfunctional PCDH19 allele) neurons. To ensure appropriate levels of gene expression, females undergo X-inactivation in which one $\mathrm{X}$ chromosome is silenced. Females heterozygous for $\mathrm{PCDH19}$ mutations will therefore also exhibit somatic mosaicism due to ranh.cooper@uq.edu.au 


\section{Science}

\section{The Weyl side of ultracold matter}

Nathan GoldmanTarik Yefsah

Science, 372 (6539), • DOI: 10.1126/science.abg0892

\section{View the article online}

https://www.science.org/doi/10.1126/science.abg0892

Permissions

https://www.science.org/help/reprints-and-permissions 